\title{
Rheumatology Mini Focus
}

“...our understanding of the cellular and molecular processes underlying arthritis have driven the development of new therapeutics."

The term 'rheumatic disease' refers to conditions affecting the joint and associated tissues. As such, it covers an incredibly broad area from the biomechanics of bone to the molecular biology of immune function. Pathologies within this field have been divided into degenerative arthropathies (osteoarthritis $[\mathrm{OA}]$ ), inflammatory arthropathies (rheumatoid arthritis [RA], spondyloarthropathies and others) systemic/connective tissue diseases (systemic lupus erythematosis, Sjogren's syndrome and others), bone disease (osteoporosis and others), congenital disorders (Marfan's Syndrome and others) and soft tissue injuries (tennis elbow, fibromyalgia and others). As might be expected, therapies vary widely, depending on the underlying pathology defining the disease state. For OA, the alleviation of pain is still the major focus of clinical trials [1]. Many clinicians feel that this is a double-edged sword (especially for OA of the knee), as decreased pain will most likely result in increased behaviors leading to further damage to the structure. Stem cell-based therapies are undergoing clinical trials and may define the future direction of therapeutic interventions [2]. However, issues of reliability are far from being solved. RA therapies underwent a transformative period in the 1990 s with the introduction of the first biologics, which inhibited cytokine function. In recent years, that strategy is still ongoing with the recent approval of the IL- 6 inhibitor, tocilizumab, for use in the USA in 2010 [3]. Other inflammatory arthropathies, such as ankylosing spondylitis and psoriatic arthritis, share aspects of pathology and recent data indicates that they are also responding relatively well to these therapies [4]. Cell-based therapies are also being examined. In particular, the programming of dendritic cells to become tolerogenic has been explored in the context of animal models of RA [5] and clinical trials are underway (NCT01352858). New targets are also being explored such as the JAKs. Pfizer's JAK inhibitor, tofacitinib, has successfully passed Phase III trials and is expected to be approved in the coming months [6]. Given the contribution of antibodies to the pathology of systemic lupus erythematosis, B cells remain the primary therapeutic target. Surprisingly, clinical trials with many of the biologics have met with failure when attempting to treat this disease [7]. The failure of Rituximab ${ }^{\mathrm{TM}}$ was especially surprising, however, some feel that the fault lies more with clinical trial design, rather than with the drug itself and that Rituximab deserves another opportunity. Recent data has identified the BLyS pathway as a good target given its role in B-cell proliferation and survival. The BLyS inhibitor, belimumab, has recently passed Phase III trials [8] and has won US FDA approval. Other therapeutics targeting the BLyS pathway are in various stages of clinical trials. For osteoporosis, the osteoclast has proved to be a worthy target. Osteoclast differentiation is dependent on the Receptor activator of $\mathrm{NF}-\kappa \mathrm{B}$ ligand and targeting this protein with the antibody, denosumab, has proven successful [9] and was approved for use in osteoporosis in 2010. In summary, advances in our understanding of the cellular and molecular processes underlying arthritis have driven the development of new therapeutics.

\section{In this issue}

In this special issue of Future Medicinal Chemistry, the field of rheumatology is featured with a series of timely reviews and editorials. Melamud points out that OA bears some similarities with RA and, thus, drugs for RA should not be discounted in their application to OA [10]. Particularly interesting is the observation of the linkage between pain signaling and inflammation. The inference is that pain blockade may also target inflammation-mediated damage. This concept is further explored in a piece by Seibel [11]. Serotonin mediates both CNS circuitry and functions as a peripheral autacoid. While $5 \mathrm{HT}_{2}$ receptor antagonists have not shown much promise, the $5 \mathrm{HT}_{3}$ receptor antagonists appear to work as well as corticosteroids but without the Cushingoid side effects. These compounds were originally designed to

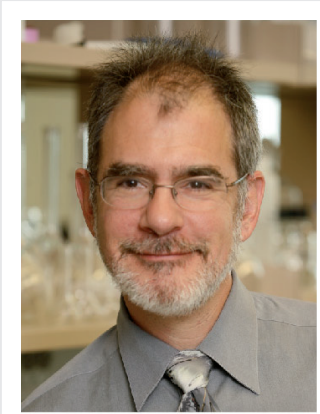

Robert I Scheinman

Department of Pharmaceutical Sciences, University of Colorado Denver Anschutz Medical Campus, 12850 East Montview Boulevard, Aurora, CO 80045, USA E-mail: robert.scheinman@ ucdenver.edu 
treat nausea caused by chemotherapeutic agents. Head-to-head clinical trials will be necessary to accurately assess the relative utility of these compounds in the arthropathies. Yet another link between pain and inflammation is forged by Dunn and colleagues in their discussion of cannabinoids as potential therapeutics in RA [12]. The endocannabinoid system consists of several G-protein-linked receptors that modulate appetite, mood, and pain sensation. In addition, certain non-psychotropic components of Cannabis sativa, the cannabidiols, appear to have antioxidant, anti-inflammatory and neuroprotective properties, which may be independent of the endocannabinoid receptors [13]. These signaling molecules are largely prostaglandin-like arachadonic acid derivatives. Interestingly, cannabinoids, when administered in animal models of RA, appear to have chondroprotective effects. Understanding the physiology underlying these observations may provide us with important new therapeutic targets. Extending the concept of lipid-derived signaling molecules, Lai and coworkers discuss the role of sphingosine signaling in RA and the potential for this pathway to provide novel signaling targets [14]. Interest in sphingosine-1-phosphate (S1P) was piqued by the observation that it played an important role in cellular proliferation [15]. Since then, the field has exploded with the understanding that S1P contributes to such diverse processes as cytokine secretion, lymphocyte trafficking, cell growth and survival. This occurs both through the binding of extracellular S1P to a family of five G-protein-linked receptors and through the actions of intracellular S1P as an intracellular second messenger. Two different kinases have been identified that phosphorylate sphingosine to create S1P and these kinases appear to have markedly different effects on disease progression in RA. Another class of signaling molecules that have become of interest to the field of rheumatology are the secretory products of white adipose tissue; the adipokines. In this issue, Evans and colleagues discuss the adipokine known as visfatin and its role in RA [16]. Visfatin inhibitors have been developed to treat certain cancers and recently these therapeutics are being examined in clinical trials for the treatment of RA. Finally, Scheinman discusses the issue of negative feedback loops in chronic autoimmune and inflammatory conditions such as RA [17]. In healthy individuals, the immune response is carefully controlled via timed activation of specific negative regulators. For example, the expansion and subsequent collapse of the clonal T-cell effectors mediating some immune response is controlled via one signal, which activates both proliferative and apoptotic responses with different kinetics [18]. In patients suffering from chronic autoimmune disease, these processes have lost their timing and one sees both effector and control signals acting at the same time. Genetic experiments in animal models have shown that these negative feedback processes play an important role in tempering disease as their removal significantly accelerates disease progression [19]. Given that these processes share signaling molecules with pathological circuits, it is to be expected that therapeutics will inhibit both types of circuits indiscriminately.

We hope that the articles published in this special issue provide valuable insights into the current contributions of medicinal chemistry efforts to rheumatic disease research.

\section{Financial \& competing interests disclosure}

The author has no relevant affliations or financial involvement with any organization or entity with a financial interest in or financial conflict with the subject matter or materials discussed in the manuscript. This includes employment, consultancies, honoraria, stock ownership or options, expert testimony, grants or patents received or pending, or royalties.

No writing assistance was utilized in the production of this manuscript.

\section{References}

1 Felson DT. Osteoarthritis in 2010: new takes on treatment and prevention. Nat. Rev. Rheumatol. 7(2), 75-76 (2011).

2 Jiang YZ, Zhang SF, Qi YY, Wang LL, Ouyang HW. Cell transplantation for articular cartilage defects: principles of past, present, and future practice. Cell Transplant. 20(5), 593-607 (2011).
3 Woodrick RS, Ruderman EM. Interleukin 6 inhibition - RA and beyond. Bull. NYU Hosp. Jt Dis. 69(3), 225-229 (2011).

4 Tak PP, Kalden JR. Advances in rheumatology: new targeted therapeutics. Arthritis Res. Ther. 13(Suppl. 1), S5 (2011).

5 Stoop JN, Robinson JH, Hilkens CM. Developing tolerogenic dendritic cell therapy for rheumatoid arthritis: what can we learn from mouse models? Ann. Rheum. Dis. 70(9), 1526-1533 (2011).

6 Garber K. Pfizer's JAK inhibitor sails through Phase 3 in rheumatoid arthritis. Nat. Biotechnol. 29(6), 467-468 (2011).

7 Eisenstein M. Approval on a knife edge. Nat. Biotechnol. 30(1), 26-29 (2012).

8 Espinosa G, Cervera R. Belimumab, a BLyS-specific inhibitor for the treatment of 
systemic lupus erythematosus. Drugs Today (Barc.) 46(12), 891-899 (2011).

9 Tsourdi E, Rachner TD, Rauner M, Hamann C, Hofbauer LC. Denosumab for bone diseases: translating bone biology into targeted therapy. Eur. J. Endocrinol. 165(6), 833-840 (2011).

10 Malemud CJ. Targeted drug development for arthritis. Future Med. Chem. 4(6), 701-703 (2012).

11 Seidel MF. Local injections of serotonin type-3 receptor antagonists as a therapeutic option in rheumatology. Future Med. Chem. 4(6), 705-707 (2012).

12 Dunn SL, Wilkinson JM, Crawford A, Le Maitre CL, Bunning RAD. Cannabinoids: novel therapies for arthritis? Future Med. Chem. 4(6), 713-725 (2012).
13 Booz GW. Cannabidiol as an emergent therapeutic strategy for lessening the impact of inflammation on oxidative stress. Free. Radic. Biol. Med. 51(5), 1054-1061 (2011).

14 Lai W-Q, Chia FLA, Leung BP. Sphingosine kinase and sphingosine-1-phosphate receptors: novel therapeutic targets of rheumatoid arthritis? Future Med. Chem. 4(6), 727-733 (2012).

15 Zhang H, Desai NN, Olivera A, Seki T, Brooker G, Spiegel S. Sphingosine-1phosphate, a novel lipid, involved in cellular proliferation. J. Cell Biol. 114(1), 155-167 (1991).

16 Nowell M, Evans L, Williams A. PBEF/ NAMPT/visfatin: a promising drug target for treating rheumatoid arthritis? Future Med. Chem. 4(6), 751-769 (2012).
17 Scheinman RI. Pleiotropic targets: the problem of shared signaling circuitry in rheumatoid arthritis disease progression and protection. Future Med. Chem. 4(6), 735-750 (2012).

18 Krammer PH, Arnold R, Lavrik IN. Life and death in peripheral T cells. Nat. Rev. Immunol. 7(7), 532-542 (2007).

19 Williams-Skipp C, Raman T, Valuck RJ, Watkins H, Palmer BE, Scheinman RI. Unmasking of a protective tumor necrosis factor receptor I-mediated signal in the collagen-induced arthritis model. Arthritis Rheum. 60 (2), 408-418 (2009). 